\title{
Analysis of recurrence factors associated with conservative surgery for ovarian-type endometriosis
}

\author{
Gu Huang", Xiang Fan", Pengfeng Zhu \\ Department of Gynecology and Tumors, Changzhou Maternal and Child Health Care Hospital, Affiliated with Nanjing Medical University, \\ Changzhou, China \\ Contributions: (I) Conception and design: G Huang; (II) Administrative support: P Zhu; (III) Provision of study materials or patients: P Zhu; (IV) \\ Collection and assembly of data: G Huang, X Fan; (V) Data analysis and interpretation: P Zhu; (VI) Manuscript writing: All authors; (VII) Final \\ approval of manuscript: All authors. \\ "These authors contributed equally to this work. \\ Correspondence to: Pengfeng Zhu. Department of Gynecology and Tumors, Changzhou Maternal and Child Health Care Hospital, Affiliated with \\ Nanjing Medical University, \#16 Dingxiang Road, Changzhou 213000, China. Email: zpf68999@163.com.
}

Background: To investigate the factors associated with postoperative recurrence in patients with ovariantype endometriosis (EMS) who were treated with conservative surgery and to provide ideas for preventing disease recurrence in advance of treatment.

Methods: The study included data from 289 inpatients with ovarian-type EMS who underwent conservative laparoscopic surgery in the Department of Gynecology, Changzhou Maternal and Child Health Care Hospital, Nanjing Medical University, from 2018 to 2020. We collected general clinical data, postoperative recurrence, drug treatment, and follow-up information. The factors associated with recurrence were analyzed using SPSS 26.0 software.

Results: A total of 289 patients were included in this study, including 49 patients with recurrence and 240 without recurrence. In the univariate analysis of recurrence, revised American Fertility Society (r-AFS) score, preoperative dysmenorrhea, EMS fertility index (EFI) score, combined myoma, and postoperative pregnancy affected postoperative recurrence of patients. The critical value of r-AFS was 70 according to the subject receiver operating characteristic curve (ROC), and the Youden index was 0.513 . R-AFS $>70$ had a sensitivity of $57.1 \%$, specificity of $94.2 \%$, the positive predictive value of $66.7 \%$, the negative predictive value of $91.5 \%$, and diagnostic accuracy of $87.9 \%$ for the diagnosis of recurrent ovarian endometrioid cysts. Using multifactorial binary logistic regression, results showed that r-AFS score $>70$ [odd ratio $(\mathrm{OR})=1.042$; 95\% confidence interval (CI): 1.028-1.055; $\mathrm{P}<0.05$ ], myoma $(\mathrm{OR}=2.995 ; 95 \%$ CI: 1.429-6.275; $\mathrm{P}<0.05)$, and preoperative dysmenorrhea $(\mathrm{OR}=1.994 ; 95 \% \mathrm{CI}: 1.071-3.713 ; \mathrm{P}<0.05)$ were risk factors for recurrence. EFI score $(\mathrm{OR}=0.785$; 95\% CI: 0.661-0.931; $\mathrm{P}<0.05)$, postoperative pregnancy (OR =0.349; 95\% CI: $0.132-$ $0.920 ; \mathrm{P}<0.05)$ were protective factors for recurrence. The differences were statistically significant, and the remaining differences were not statistically significant.

Conclusions: Preoperative r-AFS level, preoperative dysmenorrhea, and combined myoma are risk factors for postoperative recurrence, so patients with an r-AFS score over 70 should be more alert to possible recurrence. Moreover, EFI score and postoperative pregnancy are protective factors for postoperative recurrence. Postoperative patients should routinely take medication for prevention, and patients with the intention to become pregnant should be encouraged to conceive as soon as possible to reduce recurrence.

Keywords: Ovarian-type endometriosis (ovarian-type EMS); recurrence; risk factors; protective factors; conservative laparoscopic surgery

Submitted Dec 23, 2021. Accepted for publication Feb 14, 2022.

doi: $10.21037 /$ atm-22-189

View this article at: https://dx.doi.org/10.21037/atm-22-189 


\section{Introduction}

Endometriosis (EMS) is a common gynecological disease in women. Its incidence is increasing year by year. The characteristics of frequent recurrence, infertility, and pain are a serious burden to women's psychology and physiology. Ovarian EMS-like cysts are a major type of pathology of EMS. The current treatment for EMS is surgery and medication (1). Indications for drug treatment: (I) ovarian endometriosis cyst $<4 \mathrm{~cm}$ in diameter; (II) pelvic pain. The diagnosis of ovarian endometriosis cysts should be clear and laparoscopic surgery should be performed if other ovarian masses cannot be excluded. Indications for surgical treatment: (I) ovarian endometriosis cysts $\geq 4 \mathrm{~cm}$ in diameter; (II) combined infertility; (III) ineffective pain medication. However, after surgery, the recurrence rate is high, and drug therapy has limitations. Although many surgical modalities have emerged, such as ultrasoundguided cyst puncture and ovarian cyst puncture combined with drug infusion therapy, they require no adhesions or breakage of the lesion and are limited to a single lesion, although they may be less invasive (2). Therefore, laparoscopic cyst debridement under direct vision is still the first choice for diagnosing and treating EMS. In this study, we retrospectively analyzed the clinical data and followup information of patients with ovarian-type EMS treated with laparoscopic ovarian cyst debulking to investigate the factors associated with recurrence of EMS after surgery and to provide a theoretical basis for the prevention of EMS recurrence and advance management. If the influencing factors affecting the recurrence of EMS can be confirmed in the follow-up analysis, individualized medical treatment could be provided for patients with possible recurrence to alleviate physical and psychological pain and reduce the medical burden. The objectives of this study were to evaluate factors associated with EMS recurrence that affect EMS, such as age, hospital days, preoperative combined dysmenorrhea, combined infertility, combined adhesions, combined adenomyosis or myomas, combined pelvic ectasia, the maximum diameter of ovarian cysts, unilateral or bilateral cysts, revised American Fertility Society (r-AFS) score and staging, least function (LF) score, EMS fertility index (EFI) score, postoperative gonadotropinreleasing hormone agonist (GnRH-a) treatment duration, and postoperative pregnancy, all of which were compared with non-recurrent patients. The r-AFS score was based on the size and depth of peritoneal and ovarian lesions, the extent and degree of ovarian and fallopian tube adhesions, and the degree of rectal trap closure. There are 4 stages: Stage I (microscopic lesions): 1-5 points; Stage II (mild): 6-15 points; Stage III (moderate): 16-40 points; Stage IV (severe): $>40$ points. The EFI score is mainly used to predict spontaneous pregnancy after laparoscopic staging in patients with endometriosis combined with infertility. The higher the score, the higher the probability of pregnancy. The LF score refers to the unilateral (left or right) score of each of the three sites of the fallopian tube, umbilical tube, and ovary. The lowest unilateral score was obtained from both sides and the LF score was obtained by summing the two scores, which was included in the final statistics. We hypothesized that there are factors that influence recurrence of EMS and that we can predict recurrence of EMS by these factors. We compared the r-AFS, LF, and EFI scores and calculated the r-AFS threshold for EMS recurrence by receiver operating characteristic curve (ROC).

We present the following article in accordance with the STARD reporting checklist (available at https://atm. amegroups.com/article/view/10.21037/atm-22-189/rc).

\section{Methods}

\section{Patients}

For the diagnosis of ovarian-type EMS, ultrasonography is sensitive. Transvaginal ultrasound is preferred. For those who are not suitable for transvaginal ultrasound (e.g. no sexual history), abdominal ultrasound or transrectal ultrasound could be considered. We reviewed 662 patients with ovarian-type EMS who underwent laparoscopic ovarian cyst debulking in the gynecology department of Changzhou Maternal and Child Health Care Hospital (affiliated with Nanjing Medical University) from 2018 to 2020. We excluded patients with incomplete information, lost visits, and those who did not want to cooperate with a follow-up visit. Finally, 289 patients were included in this study. The medical records and follow-up information of these 289 patients were then retrospectively reviewed by one of the authors. This study was approved by the Ethics Committee of Changzhou Maternal and Child Health Care Hospital, Affiliated with Nanjing Medical University (No. 2020110). This study was conducted in accordance with the Declaration of Helsinki (as revised in 2013). Written informed consent was obtained from all participants. 


\section{Inclusion criteria}

A patient was included in the study if she satisfied the following criteria: (I) aged 18 to 45 years at the time they underwent surgery; (II) surgery was laparoscopic ovarian cyst debulking, which is a fertility-preserving surgery that is done without removing fallopian tubes and ovaries; (III) complete clinical history data and follow-up information were available; and (IV) postoperative follow-up time was more than 12 months.

\section{Exclusion criteria}

Patients were excluded if they satisfied the following criteria: (I) patients treated with radical surgery or who had gynecologic malignancies or with other malignancies; (II) patients with incomplete medical records or lost visits or who were unwilling to cooperate with a follow-up visit; (III) patients who had laparoscopic intermediate open surgery; or (IV) patients with pelvic tuberculosis, ovulation disorders, genital malformations, and other causes of infertility.

This study was conducted at the Department of Gynecology and Tumors, Changzhou Maternal and Child Health Care Hospital, Affiliated with Nanjing Medical University, Changzhou, China. We reviewed our database of patients who underwent laparoscopic ovarian cyst debulking in 2018-2020 and who were willing to cooperate with our follow-up. The first author of this study evaluated and screened all patients undergoing laparoscopic ovarian cyst debulking in 2018-2020 in the database for study eligibility.

\section{Patient treatment modalities}

Laparoscopic surgery was only feasible after all patients were excluded from contraindications related to laparoscopic surgery. After general anesthesia, the patient was placed into the laparoscope for exploration. Then, tissue adhesions were separated, an incision was made in the ovarian cortex, the tissue boundary between the cyst and the ovary was determined, and the cyst envelope was peeled completely with a combination of blunt and sharp methods. The cyst fluid was aspirated from the rupture if the cyst ruptured. The rupture could be slightly enlarged if there was no rupture. A small incision was made electrically in the non-vascular area of the ovarian cyst surface to aspirate the cyst fluid and grasp the cyst wall with grasping forceps to dissect it bluntly. The cyst was separated preferably by a reverse carpet-type method. The ovarian wound could be thermostatically stopped with bipolar accurate electrocoagulation, and the remaining ovarian tissue was sutured with absorbable thread to reconstruct the ovary. The cyst wall tissue was sent to the pathology department. Pelvic peritoneal lesions were electrocoagulated or excised. Other combined diseases, such as uterine fibroids, were treated symptomatically if there were other combined diseases. The pelvic cavity should be repeatedly irrigated, and sodium hyaluronate gel should be injected to prevent adhesions.

Postoperative medications are GnRH-a for 3-6 courses of treatment. Laparoscopic surgery is the first treatment choice for ovarian coeliacs, and postoperative treatment with the drug GnRH-a can effectively delay the recurrence of the disease.

\section{Subject data collection and follow-up}

We collected clinical records to record patients' dysmenorrhea, menstrual status, surgical history of ovarian EMS cysts, hospitalization time, r-AFS score and staging, unilateral/bilateral, maximum diameter of cysts, infertility status, adhesions, presence of comorbidities and postoperative medications, and postoperative pregnancy. The follow-up mode mainly included telephone follow-up and outpatient follow-up. The follow-up included checking patients' main clinical symptoms, postoperative medication, and whether they have recurrence or pregnancy. The interval of follow-up was greater than 12 months.

\section{Relapse criteria}

A patient was considered to have relapsed if they had the following: (I) recurrence of symptoms; (II) reappearance of adnexal masses; (III) higher than normal serum CA125 levels; and (IV) ultrasonographic confirmation of recurrence based on the criteria proposed by Kupfer et al. [1992] (3). Two clinicians with 12 and 10 years of experience in the diagnosis of EMS in the gynecologic oncology department made all recurrence diagnoses based on the above criteria. We excluded patients whose data were incomplete or who met any of the exclusion criteria, and 373 patients were excluded. The recurrence rate of postoperative chocolate cysts was related to the duration of follow-up. We selected patients who took the operation 12 months ago to ensure the recurrence of the disease. We selected patients who were operated on in 2018-2020 and finally included 289 patients. 


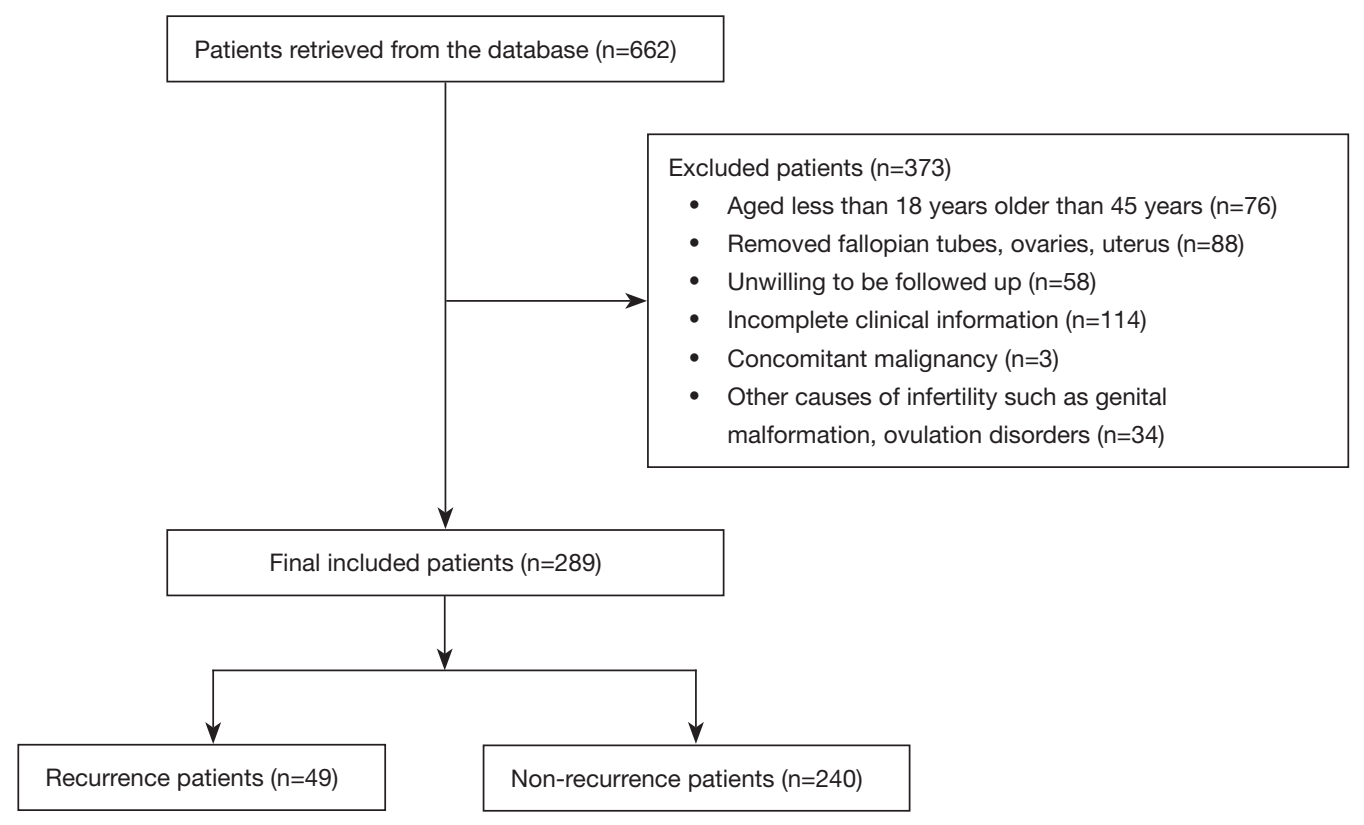

Figure 1 The flow of selecting the final participants.

\section{Statistical analysis}

We applied SPSS 26.0 software (International Business Machines Corp, USA) for statistical analysis. The measurement data were expressed as mean \pm standard deviation (SD) or median (P25-P75), the median is the number in the middle of a set of data arranged in order, and the count data were expressed as percentages or rates. Two independent samples were compared by the rank-sum test (Mann-Whitney U Test) for two groups of measurement data. Multiple groups were compared by the rank-sum test (Kruskal-Wallis Test) between multiple groups of measurement data. The chi-square test was used to compare rates. We fitted logistic regression models, and the OR values and their $95 \%$ confidence intervals (CIs) were used to evaluate the association between relevant clinical indicators and the strength of the association between EMS recurrence by OR and its $95 \% \mathrm{CI}$. All tests were performed using a two-sided test, and differences were considered statistically significant at $\mathrm{P}<0.05$.

\section{Results}

\section{Participant flow}

A total of 622 patients underwent laparoscopic ovarian cyst debulking during 2018-2020. Figure 1 shows the reasons for excluding patients. After exclusions, 289 patients were included in the study.

\section{General clinical information}

A total of 289 patients were included in this study, of whom $49(17.0 \%)$ were recurrent and $240(83.0 \%)$ were nonrecurrent, with a mean age of $34.71 \pm 5.783$ years, and a mean hospital stay of $7.90 \pm 1.733$ days. The 289 patients, $184(63.7 \%)$ had combined dysmenorrhea, 75 (30.0\%) had combined infertility, all had a maximum ovarian diameter of $6.13 \pm 2.75 \mathrm{~cm}, 204(70.6 \%)$ had unilateral ovarian cysts, 85 (29.4\%) had bilateral ovarian cysts, 226 (78.2\%) had combined adhesions, their mean r-AFS score was $39.71 \pm 26.118,233(80.6 \%)$ were stage III-IV patients, they had a mean EFI score of $5.43 \pm 1.735,30(10.4 \%)$ had combined adenomyosis, 57 (19.7\%) had combined myoma, $45(15.6 \%)$ had combined pelvic ectasia, 129 (44.6\%) had postoperative GnRH-a treatment for 3 months, 48 (16.6\%) had postoperative GnRH-a treatment for 6 months, and 64 (22.1\%) had postoperative pregnancy, as shown in Table 1.

\section{Single-factor analysis of recurrence}

The r-AFS score, preoperative dysmenorrhea, r-AFS staging, EFI score, combined myoma, and postoperative pregnancy affected postoperative recurrence $(\mathrm{P}<0.05)$, and the difference was statistically significant. In contrast, age, 
Table 1 General clinical factors

\begin{tabular}{|c|c|}
\hline Factors & Numbers \\
\hline Recurrence, n (\%) & $49(17.0)$ \\
\hline Non-recurrence, n (\%) & $240(83.0)$ \\
\hline Age (year) & $34.71 \pm 5.783$ \\
\hline Hospitalization days & $7.90 \pm 1.733$ \\
\hline Preoperative dysmenorrhea, n (\%) & $184(63.7)$ \\
\hline Infertility, n (\%) & $75(30.0)$ \\
\hline Maximum diameter of the ovary & $6.13 \pm 2.75$ \\
\hline Unilateral ovary, n (\%) & $204(70.6)$ \\
\hline Bilateral ovarian cysts, $\mathrm{n}(\%)$ & $85(29.4)$ \\
\hline Combined adhesions, n (\%) & 226 (78.2) \\
\hline r-AFS score & $39.71 \pm 26.118$ \\
\hline r-AFS stage III-IV, n (\%) & $233(80.6)$ \\
\hline EFI score & $5.43 \pm 1.735$ \\
\hline Combined adenomyosis, n (\%) & $30(10.4)$ \\
\hline Combined myxoma, n (\%) & $57(19.7)$ \\
\hline Combined pelvic heterotopia, $\mathrm{n}(\%)$ & $45(15.6)$ \\
\hline \multicolumn{2}{|l|}{ Postoperative GnRH-a therapy, n (\%) } \\
\hline 3 months & $129(44.6)$ \\
\hline 6 months & $48(16.6)$ \\
\hline Postoperative pregnancy, n (\%) & $64(22.1)$ \\
\hline
\end{tabular}

r-AFS, revised American Fertility Society; EFI, EMS fertility index; $\mathrm{GnRH}-\mathrm{a}$, gonadotropin-releasing hormone agonist.

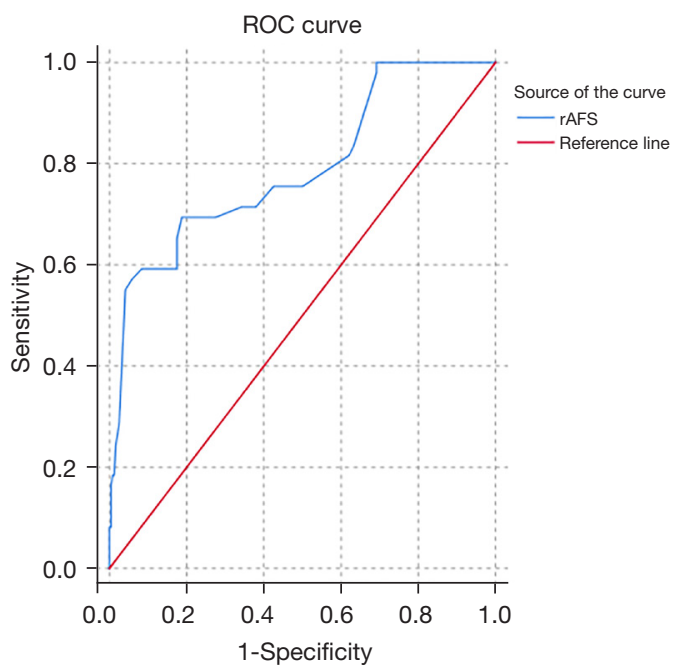

Figure 2 ROC curve. ROC, receiver operating characteristic; r-AFS, revised American Fertility Society. hospital days, combined infertility, combined adhesions, LF score, combined adenomyosis, combined pelvic ectasia, and postoperative GnRH-a treatment had no significant effect on postoperative recurrence $(\mathrm{P}>0.05)$. Results are shown in Table 2. The critical value of the r-AFS score was 70 according to the subject receiver operating characteristic curve (ROC). The area under the ROC curve was 0.790 and $\mathrm{P}<0.001$, with a $95 \%$ CI of $0.713-0.866$ and a Youden index of 0.513 (Figure 2).

Taking the r-AFS score $>70$ as the diagnostic cut-off point, 28 of 49 relapsed patients were diagnosed as relapsed, 21 were diagnosed as non-relapsed, 14 of 240 nonrelapsed patients were diagnosed as relapsed, and 226 were diagnosed as non-relapsed (Table 3).

A score of r-AFS $>70$ had a sensitivity of $57.1 \%$, a specificity of $94.2 \%$, a positive predictive value of $66.7 \%$, a negative predictive value of $91.5 \%$, and a diagnostic accuracy of $87.9 \%$ for the diagnosis of recurrent ovarian endometrioid cysts.

\section{Multifactorial analysis affecting recurrence}

Binary logistic regression results showed that r-AFS score $>70(\mathrm{OR}=1.042 ; 95 \%$ CI: $1.028-1.055 ; \mathrm{P}<0.05)$, combined myoma $(\mathrm{OR}=2.995 ; 95 \% \mathrm{CI}: 1.429-6.275 ; \mathrm{P}<0.05)$, and preoperative dysmenorrhea $(\mathrm{OR}=1.994$; $95 \%$ CI: 1.071 3.713; $\mathrm{P}<0.05)$ were risk factors for recurrence, while EFI score $(\mathrm{OR}=0.785 ; 95 \% \mathrm{CI}: 0.661-0.931 ; \mathrm{P}<0.05)$ and postoperative pregnancy $(\mathrm{OR}=0.349 ; 95 \%$ CI: $0.132-$ $0.920 ; \mathrm{P}<0.05)$ were protective factors for recurrence. The differences were statistically significant, and the remaining differences were not statistically significant (Table 4).

\section{Patients take postoperative medications for postoperative pregnancy.}

After patients stopped taking postoperative medication, there were $43(24.3 \%)$, and there were $21(18.75 \%)$ pregnancies in patients who did not take postoperative medication, with no statistically significant difference $\left(\chi^{2}=1.223 ; \mathrm{P}=0.269\right)$. After stopping their medication, 28 (65.1\%) pregnancies occurred within 6 months, 7 (16.3\%) pregnancies occurred during 6 months to 1 year, and $8(18.6 \%)$ pregnancies occurred during 1 to 2 years.

\section{Discussion}

EMS is a disease in which endometrial tissue (epithelial 
Table 2 Single-factor analysis of recurrence

\begin{tabular}{|c|c|c|c|c|}
\hline Factors & Recurrence $(n=49)$ & Non-recurrence $(n=240)$ & $\mathrm{Z}$ or $\chi^{2}$ & $P$ value \\
\hline Hospitalization days & 8 [7-9] & $8[7-9]$ & -0.26 & 0.979 \\
\hline Preoperative dysmenorrhea, n (\%) & $19(38.77)$ & $165(68.75)$ & 29.520 & $<0.001$ \\
\hline Infertility, n (\%) & $10(20.4)$ & $65(27.1)$ & 5.026 & 0.073 \\
\hline Unilateral ovary, n (\%) & $37(75.51)$ & $167(69.58)$ & 0.689 & 0.407 \\
\hline Bilateral ovaries, n (\%) & $12(24.49)$ & $73(30.41)$ & & \\
\hline Merging adhesions, n (\%) & $41(83.7)$ & $185(77.1)$ & 1.037 & 0.349 \\
\hline r-AFS score & 76 [32-82] & $30[16-48]$ & 6.426 & $<0.001$ \\
\hline r-AFS stage IV, n (\%) & $35(71.4)$ & $82(34.2)$ & & \\
\hline LF score & $6[3-8]$ & $7[5-8]$ & -1.138 & 0.255 \\
\hline EFI score & $5[4-6]$ & $6[5-7]$ & -2.636 & 0.008 \\
\hline Combined adenomyosis, n (\%) & $4(8.2)$ & $26(10.8)$ & 0.312 & 0.797 \\
\hline Combined myxoma, n (\%) & $19(38.8)$ & $38(15.8)$ & 13.527 & 0.001 \\
\hline Combined pelvic heterotopia, n (\%) & $9(18.4)$ & $36(15.0)$ & 0.351 & 0.523 \\
\hline \multicolumn{5}{|l|}{ Postoperative GnRH-a therapy, n (\%) } \\
\hline 3 months & $21(42.9)$ & $108(37.4)$ & 2.285 & 0.138 \\
\hline
\end{tabular}

r-AFS, revised American Fertility Society; LF, least function; EFI, EMS fertility index; GnRH-a, gonadotropin-releasing hormone agonist.

Table 3 Correlation between the diagnostic cut-off point of r-AFS score and recurrence of ovarian endometrioid cysts

\begin{tabular}{lccc}
\hline \multirow{2}{*}{ Variables } & \multicolumn{2}{c}{ r-AFS $>70$} & Total number of cases \\
\cline { 2 - 3 } & Diagnosis of non-recurrence & Diagnosis of relapse & 240 \\
Actual non-recurrence & 226 & 14 & 28 \\
Actual recurrence & 21 & 42 & 289 \\
Total number of cases & 247 & 49 \\
\hline
\end{tabular}

r-AFS, revised American Fertility Society.

or mesenchymal), which has a growth function, grows in and infiltrates the uterine cavity outside of the overlying endometrium and myometrium, with recurrent periodic bleeding and subsequent symptoms such as dysmenorrhea, chronic pelvic pain, infertility, and masses (4). In recent years, the prevalence of EMS in women of childbearing age in China has been reported in the literature to be about $10-20 \%$, with an increasing trend year by year (5). The pathogenesis of EMS is mainly considered to be the theory of menstrual blood reflux. In recent years, many scholars have analyzed the pathogenesis of EMs using endocrine factors (estrogen and progesterone and estrogen receptor 
Table 4 Recurrence multifactorial binary logistic regression

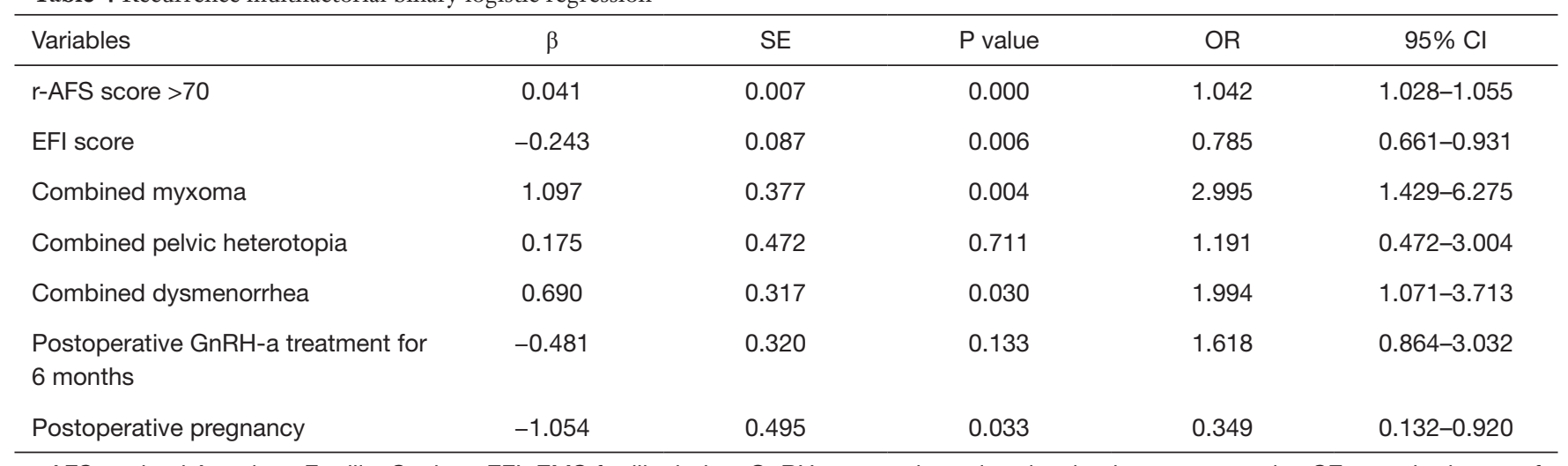

r-AFS, revised American Fertility Society; EFI, EMS fertility index; GnRH-a, gonadotropin-releasing hormone agonist; SE, standard error of estimate; OR, odds ratio; $\mathrm{Cl}$, confidence interval.

and progesterone receptor), inflammatory immune factors (tumor necrosis factor- $\alpha$, macrophage migration inhibitory factor, prostaglandin E2, and Cyclooxygenase-2), angiogenic and invasive adhesion factors (vascular endothelial growth factor, matrix metalloprotein-9), and genetic factors (6). However, the specific pathogenesis of EMS is still not fully known. Currently, laparoscopic surgery is considered the standard gold treatment for ovarian EMS $(7,8)$. However, the recurrence rate of ovarian EMS in patients without appropriate postoperative treatment can be as high as $40 \%$ within 2 years (9). Even for patients who receive appropriate postoperative treatment, the incidence of recurrence is still approximately $10 \%(10)$.

The recurrence of EMS has been a long-standing issue. Sixteen factors that may affect recurrence were analyzed by univariate, binary logistic regression in this study. r-AFS score, EFI score, preoperative dysmenorrhea, combined myoma, postoperative GnRH-a treatment, and postoperative pregnancy affected postoperative recurrence. The r-AFS score was a risk factor for postoperative recurrence. With a high r-AFS score, the lesion was more aggressive, the adhesions were heavier, and the surgery to completely remove the lesion was more difficult, therefore the recurrence rate was higher. Preoperative dysmenorrhea was a risk factor for postoperative recurrence of EMS. As an inflammatory disease, surgery can only remove the lesions visible to the naked eye, but it cannot do anything for the inflammatory milieu. Furthermore, Azuma et al. [2017] showed that, in the peritoneal fluid of patients with endo-allergy, the inflammatory factors cyclooxygenase-2 (COX-2), prostaglandin E2 (PGE2), nuclear factor- $\kappa \mathrm{B}$ $(\mathrm{NF}-\kappa \mathrm{B})$, interleukins IL-6, IL-8, and tumor necrosis factor-alpha (TNF- $\alpha$ ) were elevated (11). PGE2 was a factor in the development of dysmenorrhea, so preoperative dysmenorrhea also suggested the possibility of recurrence to some extent, which is consistent with the present study (12).

EFI scores effectively predict the occurrence of spontaneous pregnancy after surgery, and EFI scores are reliable for predicting pregnancy outcomes with nonassisted reproductive technology after EMS surgery (13). EFI scores were protective factors for recurrence of EMS in this study. The higher the patient's fertility index score, the fewer adhesions that lead to the occurrence of infertility. The higher the EFI score, the fewer adhesions, and the less inflammation and endocrine disorders that lead to the development of infertility, making postoperative recurrence relatively less likely (14). EMS is a hormonal imbalance disease similar to fibroids (15). Progesterone and estrogen signals are disrupted when endometrial tissue grows outside the uterine cavity, usually leading to progesterone resistance and estrogen dominance. One study found that symptomatic uterine fibroids and EMS have a strong connection and that combined fibroids is a risk factor for EMS recurrence, which our study also demonstrates (16).

The drugs used for the treatment of EMS include five major categories, which are non-steroidal antiinflammatory drugs, oral contraceptives, highly effective progestins, androgen derivatives, and GnRH-a. GnRH-a was mainly used for postoperative drug treatment in this study. Some studies showed that the use of GnRH-a for 6 months after surgery significantly reduced the recurrence rate (17), which is not consistent with this study. In this study, GnRH-a treatment for 3 months and GnRH-a 
treatment for 6 months had no protective effect on EMS recurrence, probably because this study is a retrospective analysis. Most of our hospitals routinely use GnRH-a to control the recurrence rate after surgery. The use of postoperative medication may only prolong the recurrence cycle, but once the medication is discontinued, the symptoms may return without improving the overall recurrence rate. In contrast, pregnancy after the combination of drugs may be a good choice for patients who want to get pregnant. Some studies have reported that the best time for pregnancy is after surgery, or within half a year after the withdrawal of combined GnRH-a, and the pregnancy rate accounts for about $50 \%$ of the 3 -year cumulative pregnancy rate, which is consistent with the present study that showed that the pregnancy rate reached $65.1 \%$ within half a year after the withdrawal of GnRH-a (18). Therefore, the recurrence of EMS can be slowed down by prolonging the use of medication or by getting pregnant as soon as possible after the surgery.

\section{Conclusions}

r-AFS score, preoperative dysmenorrhea, and combined myoma were risk factors for EMS, while EFI score and postoperative pregnancy were protective factors for EMS. An r-AFS score $>70$ had a sensitivity of $57.1 \%$, specificity of $94.2 \%$, a positive predictive value of $66.7 \%$, a negative predictive value of $91.5 \%$, and diagnostic accuracy of $87.9 \%$ in diagnosing recurrence of endometrioid cysts of the ovary, which was an independent predictor of recurrence of endometrioid cysts in the ovary. Therefore, we can evaluate the preoperative and postoperative conditions of patients to achieve more personalized diagnosis and treatment, extend the drug treatment cycle, regular review patients with a higher risk of recurrence, and reduce the use of unnecessary drugs for patients with a lower risk of recurrence to avoid excessive medical treatment. It is important to reduce unnecessary surgery, reduce patients' psychological and physiological burden, meet their fertility requirements, reduce recurrence, pay attention to follow-up, and improve their quality of life. This study only explored ovarian endometrioid cysts. However, corroborating evidence is still needed for the superficial peritoneal type and deep infiltrative EMS. The exclusion of some patients with incomplete medical records from this study may also bias the findings, and a larger sample size is needed for recurrence studies of EMS.

\section{Acknowledgments}

Funding: This project was supported partially by the major scientific research project of Changzhou Health Commission (No. ZD202121). This project had no role in the initiation or design of the study, sample collection, analysis, data interpretation, paper writing, or submission for publication. The study and the researchers were independent of the major scientific research project.

\section{Footnote}

Reporting Checklist: The authors have completed the STARD reporting checklist. Available at https://atm.amegroups. com/article/view/10.21037/atm-22-189/rc

Data Sharing Statement: Available at https://atm.amegroups. com/article/view/10.21037/atm-22-189/dss

Conflicts of Interest: All authors have completed the ICMJE uniform disclosure form (available at https://atm. amegroups.com/article/view/10.21037/atm-22-189/coif). The authors have no conflicts of interest to declare.

Ethical Statement: The authors are accountable for all aspects of the work in ensuring that questions related to the accuracy or integrity of any part of the work are appropriately investigated and resolved. This study was conducted in accordance with the Declaration of Helsinki (as revised in 2013). This study was approved by the Ethics Committee of Changzhou Maternal and Child Health Care Hospital, Affiliated with Nanjing Medical University (No. 2020110). Written informed consent was obtained from all participants.

Open Access Statement: This is an Open Access article distributed in accordance with the Creative Commons Attribution-NonCommercial-NoDerivs 4.0 International License (CC BY-NC-ND 4.0), which permits the noncommercial replication and distribution of the article with the strict proviso that no changes or edits are made and the original work is properly cited (including links to both the formal publication through the relevant DOI and the license). See: https://creativecommons.org/licenses/by-nc-nd/4.0/.

\section{References}

1. Paka C, Miller J, Nezhat C. Predictive factors and 
treatment of recurrence of endometriosis. Minerva Ginecol 2013;65:105-11.

2. Bao Y, Luo R. Treatment Measures for Postoperative Recurrence of Ovarian Endometriotic Cyst. Medical Recapitulate 2019;25:4257-60.

3. Kupfer MC, Schwimer SR, Lebovic J. Transvaginal sonographic appearance of endometriomata: spectrum of findings. J Ultrasound Med 1992;11:129-33.

4. Kennedy S, Bergqvist A, Chapron C, et al. ESHRE guideline for the diagnosis and treatment of endometriosis. Hum Reprod 2005;20:2698-704.

5. Zhang Y, Qu P. Factors associated with ovarian endometriosis malignancy and its recurrence in Chinese women. J Obstet Gynaecol 2019;39:1148-53.

6. Zhou Y. The research progress of pathogenesis and clinical applications of EMS. Journal of International Obstetrics and Gynecology 2017;44:103-7.

7. Luu TH, Uy-Kroh MJ. New Developments in Surgery for Endometriosis and Pelvic Pain. Clin Obstet Gynecol 2017;60:245-51.

8. Deckers P, Ribeiro SC, Simões RDS, et al. Systematic review and meta-analysis of the effect of bipolar electrocoagulation during laparoscopic ovarian endometrioma stripping on ovarian reserve. Int J Gynaecol Obstet 2018;140:11-7.

9. Duffy JM, Arambage K, Correa FJ, et al. Laparoscopic surgery for endometriosis. Cochrane Database Syst Rev 2014;(4):CD011031.

10. Cucinella G, Granese R, Calagna G, et al. Oral contraceptives in the prevention of endometrioma recurrence: does the different progestins used make a difference? Arch Gynecol Obstet 2013;288:821-7.

11. Azuma Y, Taniguchi F, Nakamura K, et al.

Cite this article as: Huang G, Fan X, Zhu P. Analysis of recurrence factors associated with conservative surgery for ovarian-type endometriosis. Ann Transl Med 2022;10(5):255. doi: 10.21037/atm-22-189
Lipopolysaccharide promotes the development of murine endometriosis-like lesions via the nuclear factor-kappa B pathway. Am J Reprod Immunol 2017. doi:10.1111/ aji.12631.

12. Powell AM, Chan WY, Alvin P, et al. Menstrual-PGF2 alpha, PGE2 and TXA2 in normal and dysmenorrheic women and their temporal relationship to dysmenorrhea. Prostaglandins 1985;29:273-90.

13. Garavaglia E, Pagliardini L, Tandoi I, et al. External validation of the endometriosis fertility index (EFI) for predicting spontaneous pregnancy after surgery: further considerations on its validity. Gynecol Obstet Invest 2015;79:113-8.

14. Tanbo T, Fedorcsak P. Endometriosis-associated infertility: aspects of pathophysiological mechanisms and treatment options. Acta Obstet Gynecol Scand 2017;96:659-67.

15. Marquardt RM, Kim TH, Shin JH, et al. Progesterone and Estrogen Signaling in the Endometrium: What Goes Wrong in Endometriosis? Int J Mol Sci 2019;20:3822.

16. Nezhat C, Li A, Abed S, et al. Strong Association Between Endometriosis and Symptomatic Leiomyomas. JSLS 2016;20:e2016.

17. Zheng Q, Mao H, Xu Y, et al. Can postoperative GnRH agonist treatment prevent endometriosis recurrence? A meta-analysis. Arch Gynecol Obstet 2016;294:201-7.

18. He LQ, Cai XZ, Wang Y, et al. Effect of GnRHa therapy following conservative laparoscopic surgery for endometriosis on clinical pregnant rate in patients with endometriosis-associated infertility. Nan Fang Yi Ke Da Xue Xue Bao 2018;38:596-600.

(English Language Editor: C. Mullens) 